\title{
Facing the Challenges for Europe - Research into Action: Liverpool European Congress of Obesity, May 12-15, 2013
}

\author{
John P.H. Wilding Jason C.G. Halford \\ Experimental Psychology, University of Liverpool, Liverpool, UK
}

Europe faces its greatest social and economic challenge in 70 years. We reside in a period of global economic turmoil associated with the rapid re-adjustment in the European financial landscape. The next 5 years, if not the decade, will see at best limited growth in economic terms and in all likelihood real term reductions in funding available for health provision and specifically for obesity research at least at national levels. Critical economic conditions will impact on the health of European citizens, in particular on the most vulnerable including children and the elderly in the poorest economic circumstances. It is therefore critical we continue to celebrate excellence in obesity research across scientific disciplines. The next European Congress of Obesity will seek to demonstrate the necessity of translating research into practice for the health, social and economic benefits for Europe and its citizens.

The European Congress on Obesity (ECO) will be held in the City of Liverpool, UK, from May 12-15, 2013 (www.eco2013.org). The programme aims to place obesity research at the centre of national and European policy agendas for the next decade. The scientific committee has constructed a programme more inclusive than ever before, bringing together basic science with clinical management, and behavioural and nutritional expertise with policy and intervention. Utilising cross-cutting themes, the congress will appeal to a wider possible audience than previous years, including researchers, policy makers and clinicians caring for people with obesity and related conditions. This will encourage active discussion and debate both within and across the traditional scientific, clinical and public health disciplines represented in the meeting. 
Wilding et al.: Facing the Challenges for Europe - Research into Action: Liverpoo European Congress of Obesity, May 12-15, 2013

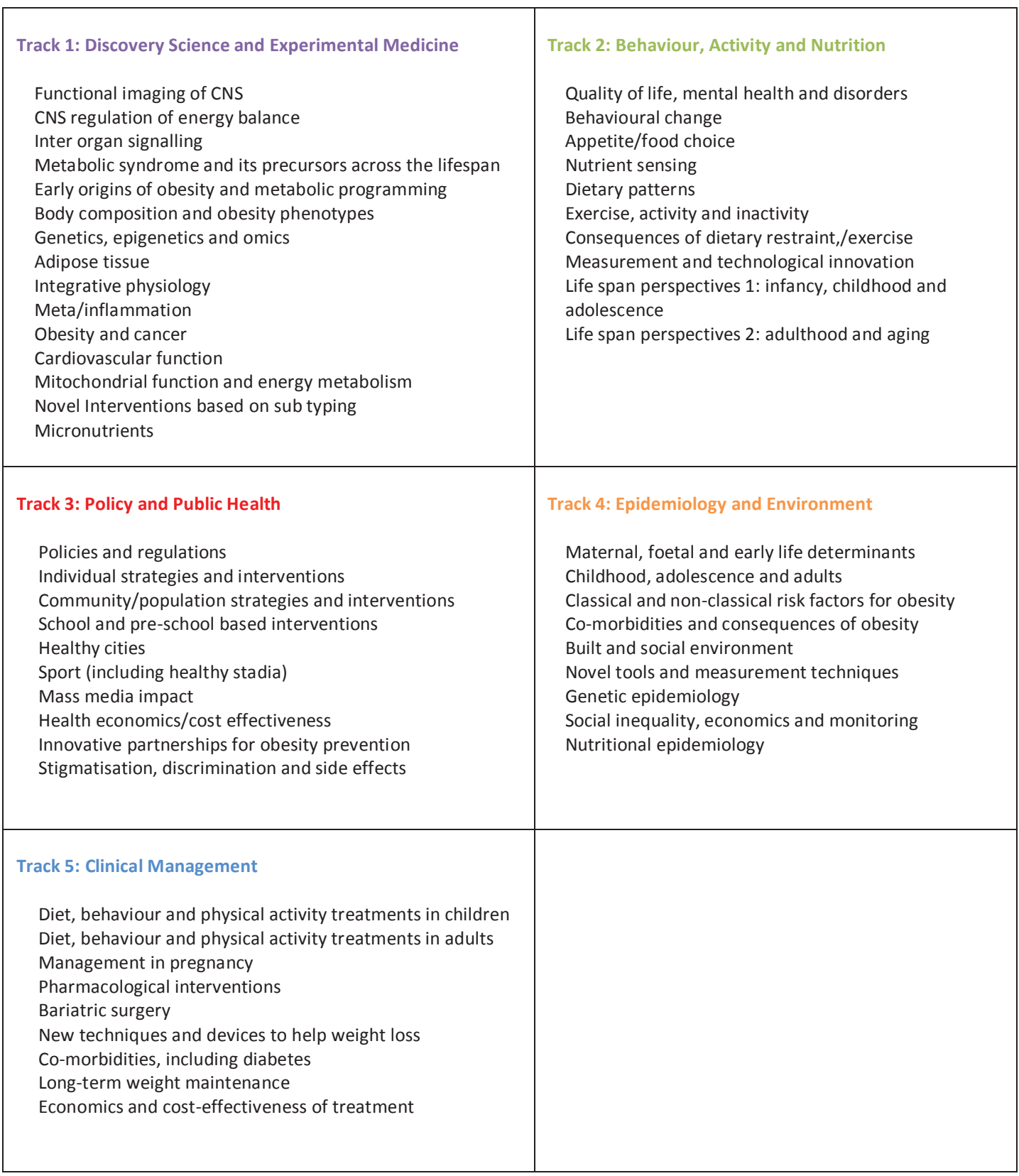

Fig. 1. Tracks and sub-topics.

\section{Scientific Programme with Wide Appeal}

The five tracks in the scientific programme will cover the important themes of Discovery Science and Experimental Medicine Behaviour, Activity and Nutrition Policy and Public Health; Epidemiology and the Environment; and Clinical Management (fig. 1). The sub-topics chosen have been designed to be comprehendible, comprehensive as well as contemporary and challenging. Within each track, keynote presentations and review lectures will be provided from international speakers recognised for the high quality of their work and presentation style. A significant number of oral presentations will also be selected from the 
Wilding et al.: Facing the Challenges for Europe - Research into Action: Liverpool European Congress of Obesity, May 12-15, 2013

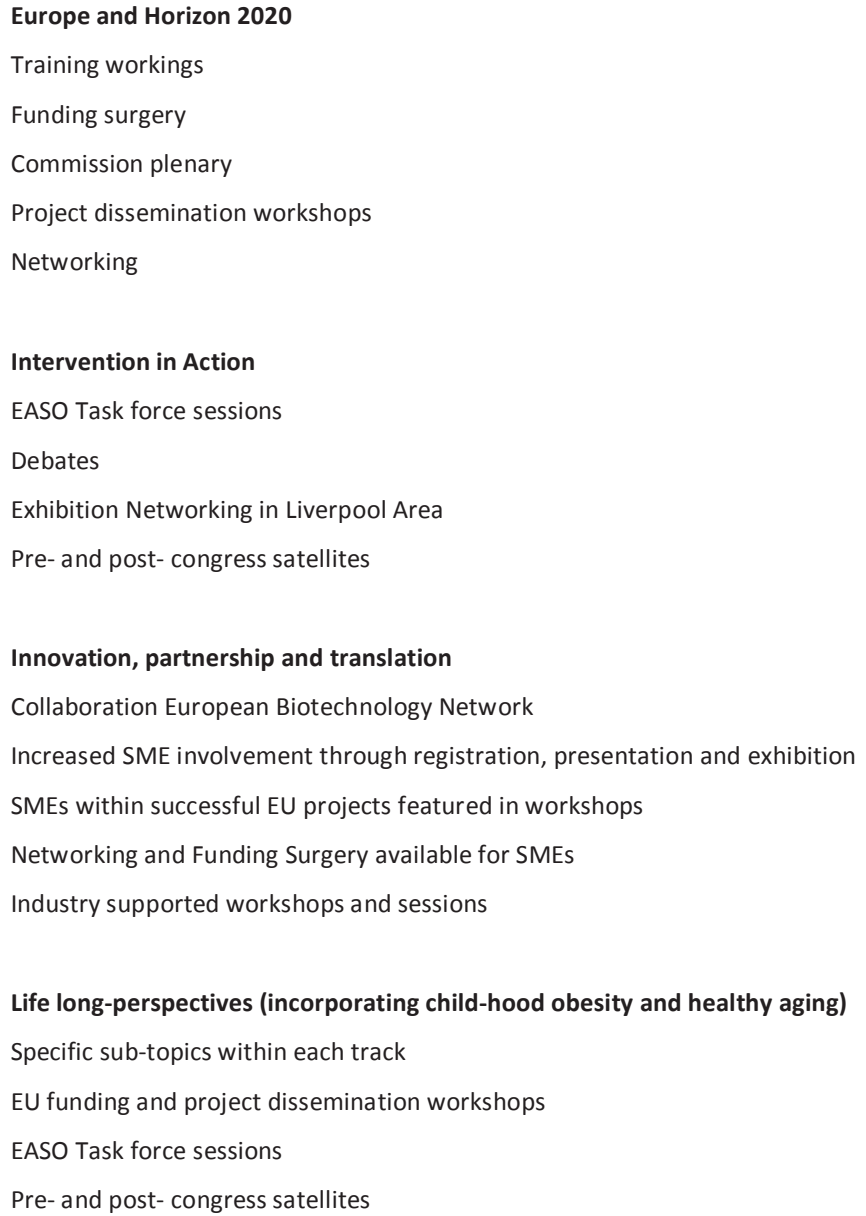

Fig. 2. Cross-cutting themes.

best abstracts submitted within each track, and the 2013 scientific committee has worked hard to provide more overall opportunities for oral presentation than at any previous ECO. In addition, outstanding research will also be featured in specifically themed poster sessions that cover distinct areas of interest based on the theme sub-topics.

\section{Cross-Cutting Themes}

The new innovation in ECO 2013 are the cross-cutting themes focused on the needs of Europe and its citizens, and designed to help obesity research address the new challenges described above (fig. 2). The aim of EASO remains clearly to support excellence in obesity science across Europe and the exchange of ideas, methodological innovations, and new advances across the obesity field. At the centre of this is interdisciplinary collaboration built on the platform of European Framework funding. The congress will encourage discussion and debate, to help delegates think in innovative ways about how to work together to develop effective interventions; and to encourage researchers in all aspects of obesity to collaborate on research ideas, particularly in relation to the next EU Framework grant scheme, Horizon 2020. 
The first cross-cutting theme, Europe and Horizon 2020, will directly address this. ECO2013, in line with EASO's current activities, will prepare and support European obesity research in the run up to formal launch of the next framework call Horizon 2020 (anticipated July 2014). Workshops in the programme will provide lunch time training sessions on i) 'How to Build an EU Project Consortium', ii) 'Partnering and Innovation', and iii) 'Funding for Collaborative Research: What We Can Expect in Horizon 2020'. In addition, the European Biotechnology Network and the University of Liverpool will provide an EU funding within the main exhibition throughout the duration of the congress. The commission will be invited to outline the key features of the new call and special workshops in a session on the European perspective: How do we plan to tackle obesity in the coming decade? Additional dissemination workshop sessions will feature key framework 6 and 7 obesity-related projects. These interactive sessions will enhance delegates' knowledge of the possibilities and potential of collaborative European research and the research base the new funding call will be building upon (current project co-ordinators interested in being involved please contact the EASO Secretariat at eco2013 @easo.org). Networking between potential participants and co-ordinators will be facilitated through these and other activities during the congress.

The second cross-cutting theme is Intervention in Action. For Europe improving the diet and health of the European citizen is critical. However, to the casual observer it often appears there is little that can be done to stem the tide of obesity. Yet across Europe, interventions of varying scales and numerous approaches with a variety of settings - offering differing degrees of support, aiming at distinct and diverse populations, and differing in outcome and proxy outcome measures - are underway. The scope and range of these interventions remains largely unknown outside the published literature, even to those with expertise in the field. For those in public health, education or in the work place tasked with tackling obesity, determining what works for whom, and even more basically, whether current interventions can be judged effective is far from straightforward. Fundamental questions still exist: Should interventions be theory or practice led? Can policy makers wait for the results of lengthy trials before translating interventions in practice?

The role of evidence in translation and delivery remains a critical issue for obesity science. ECO 2013 addresses this through plenary, track sub-topics (oral and poster sections), dedicated workshops, EASO Task Force sessions, specific debates and dedicated pre- and post-congress satellites. Specifically, a Liverpool area in the main exhibition area featuring a variety of regional interventions will act as a point of networking where researchers and health professionals from across Europe can share knowledge and exchange best practice. Specific focus will be placed on individual versus population strategies, healthy cities and stadia, and the role of partnerships. A key theme of the conference will be to look at how complex interventions that embrace public health, including the built environment and individual health-care, can work at local, regional, national and international levels to help stem the tide of the obesity epidemic. Key speakers will be addressing these questions during the conference, and the committee will particularly welcome abstract submissions in these areas.

The third cross-cutting theme is Innovation, Partnership and Translation. A key goal of the framework programme is economic growth in Europe through science and innovation, particularly through the support of small- and medium-sized enterprises (SMEs). Horizon 2020 will bring together SMEs with academia and industry to ensure that novel solutions to weight management move quickly from theory and inception, through proof-of concept and demonstration of efficacy to commercialisation. The complexity and seeming intractability of the obesity epidemic requires novel solutions that may come from unexpected areas of science, medicine and public health. It is often hard to translate even the best basic research into innovative medicines, medical devices, consumer products, monitoring or testing 
devices, dietary plans, integrated commercial programmes and viable prototype products or interventions. Moreover, accessing the funding and expertise to successfully test these approaches in small-scale studies is a considerable challenge. The conference will help address these common deficiencies, encouraging speakers, poster presenters and delegates to think beyond their immediate results towards how their ideas might translate into effective interventions, perhaps involving new ways of working and partnerships that provide synergistic benefits. Specifically, SMEs and commercial enterprises from the biotechnology sector will be encouraged to attend, present and exhibit at ECO. EU funding workshops and networking opportunities will be tailored to SME needs, and SMEs within existing European projects will feature in workshops. The management of obesity and related conditions has had a long history of partnership in the areas of pharmacology, nutrition and commercial weight management which ECO 2013 will continue to support. The congress features industry-supported workshops as well as the traditional industrysupported sessions and exhibitions.

The fourth theme is Lifelong Perspective. Healthy aging will be a key theme of Horizon 2020. Childhood obesity remains a critical concern but ECO 2013 explicitly recognises that obesity affects all ages. This conference will have extensive coverage of obesity at all stages of life from early infancy, through childhood, adolescence and early adults, through working life to middle age, retirement, and eventually old age, focusing on behavioural and situational factors as well as the biology of development and maturation. Key life events such as starting school, leaving home, marriage, starting a family, divorce or widowhood will be considered. The lifelong perspective cross-cutting theme will encourage researchers and clinicians, specialising exclusively in either paediatric or adult obesity, to think about obesity across all age groups. Healthy ageing reminds us that although obesity often has its roots in child hood, it clearly affect people of all ages, and this necessitates a life course approach. The lifelong perspective will be featured in plenary, oral and poster submissions, EASO Childhood, Management and Prevention and Public Health Task Force events, EU funding and project dissemination workshops as well as in pre- and post-congress satellites.

\section{Young Researchers}

EASO will support young researchers in a number of ways. Firstly, young researchers benefit from reduced registration fees, and for the first time, ECO 2013 will offer institute group booking discounts to allow PIs to bring a larger number of young colleagues to a major international congress. Secondly, EASO will offer travel grants (registration and hotel accommodation) via EASO National Association competitive application, meaning that young researchers from across the region will benefit. Thirdly, EASO will continue to support scientific excellence via its Young Investigator and YIU Best Thesis Awards.

To assist young researchers further, the city of Liverpool offers a wide range of low-cost accommodation options, from budget hotels to shared self-catering accommodation within the city centre. Liverpool and Manchester airports are extensively served by most European budget airlines.

Via the EASO Young Investigators United (YIU) initiative, ECO 2013 will provide networking opportunities for all young researchers, delivered via a scientific and social session as part of the main ECO 2013 programme. The YIU event will incorporate a training workshop by the European Research Council (ERC), giving practical advice on ERC grant applications. 


\section{Liverpool}

The congress has strong local support from the Liverpool City Council, which, with partners in primary health care and education (www.2010healthandwellbeing.org.uk/index. $p h p$ ) and with the Liverpool Football Club and other key sporting institution in the city, is working hard to improve the health of people that live in the city, including those with weight problems. Coupled with the serious work of science and clinical practice there will be plenty of opportunities to relax, socialise and enjoy the many attractions Liverpool has to offer (www.visitliverpool.com). 